CLNS 95/1377

MZ-TH/95-31

\title{
Sum Rules for Radiative and Strong Decays of Heavy Mesons
}

\author{
Chi-Keung Chow \\ Newman Laboratory of Nuclear Studies, Cornell University, Ithaca, NY 14853. \\ Dan Pirjoll \\ Johannes Gutenberg-Universität, Institut für Physik (THEP), \\ Staudingerweg 7, D-55099 Mainz, Germany.
}

(June 18, 2018)

\begin{abstract}
We derive two model-independent sum rules relating the transition matrix elements for radiative and strong decays of excited heavy mesons to properties of the lowest-lying heavy mesons. The sum rule for the radiative decays is an analog of the Cabibbo-Radicati sum rule and expresses the sum of the radiative widths in terms of the isovector charge radius of the ground state heavy meson. Using model-dependent estimates and heavy hadron chiral perturbation theory calculations, we show that this sum rule is close to saturation with states of excitation energies less than $1 \mathrm{GeV}$. An analog of the AdlerWeisberger sum rule gives an useful sum rule for the pionic widths of heavy excited mesons, which is used to set a model-independent upper bound on the coupling of the P-wave heavy mesons.
\end{abstract}

Typeset using REVTEX 
We present in this paper two model-independent sum rules for the photon and pion couplings to the light constituents in a heavy meson. They relate properties of the ground state such as charge radius, magnetic moment and axial coupling to matrix elements which govern the radiative and strong transitions between excited heavy meson states and the ground state. The latter parameters can be in principle extracted from experiment and in fact, a few of them have been already determined in this way. The sum rules themselves are not new, they are direct analogs of the Cabibbo-Radicati (CR) [1] and respectively Adler-Weisberger (AW) [2,3] sum rules, familiar from current algebra. However, in this new context they turn out to be considerably more predictive than in their original application, due to additional constraints on the quantum numbers of the available final states. Moreover, when considered in the large- $N_{c}$ limit, the form of the sum rules simplifies further due to the suppression of the continuum contribution. The $\mathrm{CR}$ sum rule reduces in this limit to a constituent-quark sum rule familiar from nonrelativistic quantum mechanics, connecting the charge radius of the ground state to a sum over electric dipole matrix elements between excited states and the ground state [4].

These sum rules are of interest from a phenomenological point of view, as they can be used to place constraints on the photon and pion couplings of low-lying heavy mesons to excited ones. As a sample application, we derive model-independent upper bounds on the pionic decay widths of the charmed p-wave heavy mesons with the quantum numbers of the light degrees of freedom $s_{\ell}^{\pi_{\ell}}=1 / 2^{+}$.

The Cabibbo-Radicati sum rule can be derived (we follow here the derivation given in [5 [7]) by considering the forward scattering amplitude of isovector photons of energy $\omega$ and helicity $\lambda$ on a target $B$

$$
f^{a b}(\omega, \lambda)=\frac{i}{4 \pi} \int \mathrm{d} x e^{-i q \cdot x}\left\langle B\left|\mathrm{~T}\left(J^{a} \cdot e_{\lambda}\right)(x)\left(J^{b} \cdot e_{\lambda}^{*}\right)(0)\right| B\right\rangle
$$

with $q^{0}=\omega, q^{2}=0$ and $J_{\mu}^{a}=e \bar{q} \gamma_{\mu} t^{a} q\left(t^{a}=\tau^{a} / 2\right)$. The states $|B\rangle$ are normalized noncovariantly to 1 . The scattering amplitude $f$ can be written in terms of four invariants $f_{ \pm}^{( \pm)}(\omega)$ as $\left(J_{z}\right.$ is the $z$-projection of the target spin) 


$$
\begin{gathered}
f^{a b}(\omega, \lambda)=\left\langle B\left|\frac{1}{2}\left\{t^{a}, t^{b}\right\}\left(f_{+}^{(+)}(\omega)+f_{-}^{(+)}(\omega) \lambda \omega J_{z}\right)\right| B\right\rangle \\
+\left\langle B\left|\frac{1}{2}\left[t^{a}, t^{b}\right]\left(f_{+}^{(-)}(\omega) \lambda J_{z}+\omega f_{-}^{(-)}(\omega)\right)\right| B\right\rangle .
\end{gathered}
$$

The assumption of an unsubtracted dispersion relation for $f_{-}^{(-)}(\omega)$

$$
f_{-}^{(-)}(0)=\frac{2}{\pi} \int_{0}^{\infty} \frac{\mathrm{d} \omega^{\prime}}{\omega^{\prime}} \operatorname{Im} f_{-}^{(-)}\left(\omega^{\prime}\right)
$$

in combination with the low-energy theorem [5] $\lim _{\omega \rightarrow 0} f_{-}^{(-)}(\omega)=\frac{1}{\pi}\left(-R_{V}^{2} / 6+\mu_{V}^{2} / 2\right.$ ) (for a target of isospin $\frac{1}{2}$ and spin $\frac{1}{2}$ ) leads to the final form of the CR sum rule

$$
\frac{R_{V}^{2}}{6}-\frac{\mu_{V}^{2}}{2}=\frac{1}{\pi^{2} e^{2}} \int_{0}^{\infty} \frac{\mathrm{d} \omega^{\prime}}{\omega^{\prime}}\left(2 \sigma_{1 / 2}\left(\omega^{\prime}\right)-\sigma_{3 / 2}\left(\omega^{\prime}\right)\right)
$$

On the left-hand side $R_{V}$ and $\mu_{V}$ are the isovector charge radius and magnetic moment of the target. On the right-hand side the optical theorem has been used to express $\operatorname{Im} f_{-}^{(-)}(\omega)$ in terms of the cross-sections for inclusive photoproduction by an isovector photon with $I_{z}=0$ of final states with isospin $1 / 2$ and respectively $3 / 2$.

We will take as target a pseudoscalar heavy meson with quark content $\bar{Q} u\left(I, I_{z}=\frac{1}{2},+\frac{1}{2}\right)$, denoted generically as $\left|B_{i}\right\rangle(i=u, d)$. There are a number of specific points which have to be addressed in connection with this choice. First, the sum rule (4) has been derived under the assumption that the target is a spin-1/2 particle, whereas the $\left|B_{i}\right\rangle$ meson has spin zero. However, in the heavy mass limit $m_{Q} \rightarrow \infty$ the dynamics of the heavy quark decouples from that of the light constituents. As a result, the target $\left|B_{i}\right\rangle=\frac{1}{\sqrt{2}}\left(\left|Q^{\uparrow} q_{i}^{\downarrow}\right\rangle-\left|Q^{\downarrow} q_{i}^{\uparrow}\right\rangle\right)$ can be effectively considered as a coherent superposition of polarized spin-1/2 particles.

Second, for such a target the isovector and isoscalar electromagnetic parameters are related in the $\mathrm{SU}(3)$-symmetric limit. The reason for this is that heavy mesons containing only one light quark transform according to the $\mathbf{3}$ representation of $\mathrm{SU}(3)$. The electromagnetic current transforms as an octet and there is only one way of combining $\mathbf{3}, \mathbf{8}$ and $\overline{\mathbf{3}}$ to a singlet [8 [10]. Previous applications of the CR sum rule used a proton or a pion as target, which belong to $\mathrm{SU}(3)$ octets. Therefore their isovector and isoscalar electromagnetic form-factors remain unrelated even in the $\mathrm{SU}(3)$ limit. The assumption of $\mathrm{SU}(3)$ symmetry simplifies 
very much the sum rule, and the main part of the discussion below will be restricted to this case. However, the sum rule can be modified to include $\mathrm{SU}(3)$-breaking effects and we will return to this point later.

From the above observation it follows that the elastic e.m. form-factor of a $\mathrm{B}$ meson can be written in terms of just one function $F\left(q^{2}\right)$ as $\left\langle B_{i}\left|J_{\mu}\right| B_{j}\right\rangle=e F\left(q^{2}\right) v_{\mu} \mathcal{Q}_{i j}$ with $J_{\mu}=e \bar{q} \gamma_{\mu} \mathcal{Q} q$ and $\mathcal{Q}=\operatorname{diag}\left(\frac{2}{3},-\frac{1}{3}\right)$ is the light quark charge matrix. Current conservation gives $F(0)=1$. The charge radius appearing on the l.h.s. of (田) is defined as $R_{V}^{2} / 6=d F\left(q^{2}\right) /\left.d q^{2}\right|_{q^{2}=0}$.

The isovector magnetic moment $\mu_{V}$ of the light constituents in (四) is related to

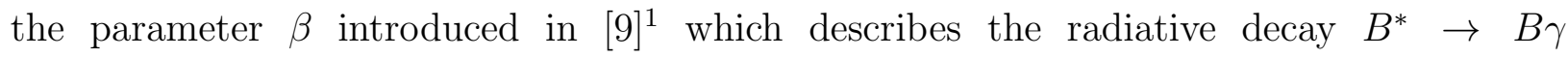
$\left\langle B(v)\left|\bar{q} \gamma_{\mu} q\right| B^{*}(v, \epsilon)\right\rangle=-i \beta \varepsilon_{\mu \nu \rho \sigma} v^{\nu} k^{\rho} \epsilon^{\sigma}$ ( $k$ is the photon momentum) as $\mu_{V}=\beta / 2$. The corresponding decay rate is equal to $\Gamma=\frac{1}{3} \alpha Q^{2} \beta^{2}|\vec{k}|^{3}$, with $Q$ the heavy quark charge in units of $e$.

Finally, another distinctive feature of our problem is the absence of resonant final states with isospin $3 / 2$. Therefore, $\sigma_{3 / 2}(\omega)$ in (四) only receives contributions from continuum states. There is one limit in which these contributions are completely suppressed, and this is the large- $N_{c}$ limit. In this case the Cabibbo-Radicati sum rule is saturated with the resonances alone, and the corresponding photoproduction cross-sections can be expressed in terms of the decay widths for the inverse process $B^{e x c} \rightarrow B \gamma$. One obtains in this way the particularly simple result

$$
\frac{R_{V}^{2}}{6}-\frac{\beta^{2}}{8}=\frac{1}{8 \alpha Q^{2}} \sum_{e x c}(2 J+1) \frac{\Gamma\left(B^{e x c} \rightarrow B \gamma\right)}{|\vec{k}|^{3}} .
$$

The summation runs over all excited states of the $|B\rangle$ meson and $J$ is the spin of each state. The form of the sum rule can be simplified by deleting the second term on the l.h.s. and extending the summation over the $B^{*}$ state as well.

\footnotetext{
${ }^{1}$ The extra (-) sign is due to the fact that we are considering heavy mesons with one heavy antiquark.
} 
It is interesting that a very similar sum rule can be obtained in the nonrelativistic constituent quark model (NRCQM) [11] (and also in atomic physics 《4) by writing

$$
\langle B|\vec{x} \cdot \vec{x}| B\rangle=\sum_{n}^{(\ell=1)}|\langle B|\vec{x}| n\rangle|^{2} .
$$

The radiative decay rate in the electric dipole approximation is given by $\Gamma_{E 1}=$ $\frac{4}{3} \alpha Q^{2}|\vec{k}|^{3}|\langle B|\vec{x}| n\rangle|^{2}$, which yields upon insertion into (6) a sum rule for the heavy meson charge radius

$$
\frac{R^{2}}{6}=\frac{1}{8 \alpha Q^{2}} \sum_{e x c}^{(\ell=1)}(2 J+1) \frac{\Gamma_{E 1}\left(B^{e x c} \rightarrow B \gamma\right)}{|\vec{k}|^{3}} .
$$

Although very similar to (5), the summation over excited states extends in (7) only over P-wave states, which are connected to the ground state by a E1 transition, whereas in the exact sum rule (5) all excited states contribute.

When the large- $N_{c}$ limit is relaxed, the integral on the right-hand side of the sum rule (4) will receive, in addition to the contributions from the excited heavy mesons (5), also contributions from continuum states like $(B \pi),\left(B^{*} \pi\right)$, etc. These can be calculated reliably in heavy hadron chiral perturbation theory [12 14], as long as the pion momentum is smaller than the chiral symmetry breaking scale $\Lambda_{\chi} \simeq 1 \mathrm{GeV}$. By keeping only the B and B* mesons in intermediate states we obtain

$$
\begin{aligned}
\sigma_{1 / 2}^{(\pi B)}(\omega) & =2 \sigma_{3 / 2}^{(\pi B)}(\omega)=\frac{\pi}{18}\left(\frac{e g \beta}{2 \pi f_{\pi}}\right)^{2} \frac{|\vec{p}|}{\omega}\left(\omega^{2}-m_{\pi}^{2}\right) \\
\sigma_{1 / 2}^{\left(\pi B^{*}\right)}(\omega) & =\frac{2 \pi}{3}\left(\frac{e g}{2 \pi f_{\pi}}\right)^{2} \frac{|\vec{p}|}{\omega}\left\{1+\frac{m_{\pi}^{2}}{\omega^{2}}+\frac{m_{\pi}^{2}}{2 \omega|\vec{p}|} \log \frac{\omega-|\vec{p}|}{\omega+|\vec{p}|}\right. \\
& \left.-\frac{1}{4} \beta \omega\left(1+\frac{m_{\pi}^{2}}{2 \omega|\vec{p}|} \log \frac{\omega-|\vec{p}|}{\omega+|\vec{p}|}\right)+\frac{1}{24} \beta^{2}\left(\omega^{2}-m_{\pi}^{2}\right)\right\}, \\
\sigma_{3 / 2}^{\left(\pi B^{*}\right)}(\omega) & =\frac{\pi}{3}\left(\frac{e g}{2 \pi f_{\pi}}\right)^{2} \frac{|\vec{p}|}{\omega}\left\{1+\frac{m_{\pi}^{2}}{\omega^{2}}+\frac{m_{\pi}^{2}}{2 \omega|\vec{p}|} \log \frac{\omega-|\vec{p}|}{\omega+|\vec{p}|}\right. \\
& \left.+\frac{1}{2} \beta \omega\left(1+\frac{m_{\pi}^{2}}{2 \omega|\vec{p}|} \log \frac{\omega-|\vec{p}|}{\omega+|\vec{p}|}\right)+\frac{1}{6} \beta^{2}\left(\omega^{2}-m_{\pi}^{2}\right)\right\}
\end{aligned}
$$

where $|\vec{p}|=\sqrt{\omega^{2}-m_{\pi}^{2}}$ is the pion momentum. The static approximation for the heavy meson has been used in deriving these expressions $m_{\pi} / m_{B} \simeq 0$. 
In these formulas $g$ is the $\mathrm{BB}^{*} \pi$ coupling in the heavy mass limit [12 14]. Experimental data on branching ratios of $\mathrm{D}^{*}$ decays [8 [10] give $0.09 \leq g^{2} \leq 0.5$ (with $90 \%$ confidence limits). We will adopt for our estimates the upper limit $g^{2}=0.5$ (note though that QCD sum rule computations suggest significantly lower values $g^{2} \simeq 0.1$ [16 [19]). As already mentioned, $\beta$ describes the $B^{*} \rightarrow B \gamma$ decay in the heavy mass limit. It has been determined simultaneously with $g$ in [8 10] such that their values are correlated: larger values for $g$ favor larger values for $\beta$. The limits quoted are $2 \leq \beta \leq 6 \mathrm{GeV}^{-1}$ 8 10]. Recent QCD sum rule and model calculations [15,16, 19] give on the other hand smaller values, around $\beta=2-3$ $\mathrm{GeV}^{-1}$ ( [19] find $\beta \simeq 1 \mathrm{GeV}^{-1}$ ). We will use in our estimates below $\beta=3-4 \mathrm{GeV}^{-1}$.

Previous experience with the Cabibbo-Radicati sum rule [23 25] suggests that the saturation region includes states with an excitation energy of the order of a few $\mathrm{GeV}$. Inserting (8) into (4) with an upper cutoff of $1 \mathrm{GeV}$ gives for the continuum contribution on the r.h.s. of the sum rule (田)

$$
\begin{aligned}
I_{\text {cont }}^{(\pi B)}+I_{\text {cont }}^{\left(\pi B^{*}\right)}+\cdots & =\left(\frac{g}{2 \pi f_{\pi}}\right)^{2} 0.036 \beta^{2}+\left(\frac{g}{2 \pi f_{\pi}}\right)^{2}(1.519-0.321 \beta)+\cdots \\
& =0.639(0.589)\left(\mathrm{GeV}^{-2}\right)+\cdots .
\end{aligned}
$$

The ellipsis stand for other continuum contributions, which are expected to be less important as they have less phase space available. The two numbers correspond to $\beta=3(4) \mathrm{GeV}^{-1}$. The pion decay constant is $f_{\pi}=0.132 \mathrm{GeV}$.

We are now in a position to discuss the numerical values of the two sides of the sum rule (四). It can be written as

$$
\begin{aligned}
& 2.161=0.321(0.846)+(0.405 \pm 0.067)+(0.811 \pm 0.135)+0.639(0.589)+\cdots .\left(\mathrm{GeV}^{-2}\right) \\
& \frac{R_{V}^{2}}{6}=\left(B^{*}\right)+\left(P_{1 / 2}\right)+\left(P_{3 / 2}\right)+(\text { continuum })
\end{aligned}
$$

On the left-hand side, the isovector charge radius $R_{V}$ has been obtained from a version of vector-meson dominance where the contributions of the two lowest $I=1\left(J^{P C}=1^{--}\right)$ vector mesons are kept $R_{V}^{2} / 6=\left(1 / m_{\rho}^{2}+1 / m_{\rho^{\prime}}^{2}\right)$. This procedure gives an elastic formfactor in agreement with the QCD counting rules at large $q^{2}$ [22]. On the right-hand side, 
the $\mathrm{B}^{*}$ contribution has been computed by including also the nonanalytic SU(3)-violating contributions obtained in [9] $\mu_{V}^{2}=\frac{1}{4}\left(\beta-\frac{g^{2} m_{K}}{4 \pi f_{K}^{2}}-\frac{g^{2} m_{\pi}}{2 \pi f_{\pi}^{2}}\right)^{2}$. The contributions of the two lowestlying P-wave states with $s_{\ell}^{\pi_{\ell}}=\frac{1}{2}^{+}, \frac{3}{2}^{+}$were computed in the dipole approximation using the wavefunctions of the ISGW model [20] with the updated parameters of the ISGW2 model [21]. In the absence of the spin-orbit interaction, responsible for the splitting of these two multiplets, their contributions to the sum rule are in the ratio $P_{1 / 2}: P_{3 / 2}=1: 2$. The errors shown correspond to the $30 \%$ accuracy expected from the model when predicting radiative decay matrix elements [21].

One obtains in this way for the r.h.s.of the sum rule $2.176 \pm 0.202(2.651 \pm 0.202) \mathrm{GeV}^{-2}$. The agreement with the l.h.s. is certainly better than one could have expected from our qualitative estimates. This strongly suggests that the $\mathrm{CR}$ sum rule is very close to saturation with the first few excited states and the continuum up to excitation energies of about $1 \mathrm{GeV}$.

We would like to make a few additional comments about the different contributions on the r.h.s. of (12).

- The nonresonant contributions have all the same sign (positive). The dominant contribution to the integral over $\omega$ comes from the $\left(\pi B^{*}\right)$ two-body state, which can be formed in a $l=0$ partial wave. Therefore this cross-section dominates in the low energy region, to which the sum rule is the most sensitive. The threshold region gives rise to a large $\log \left(g /\left(2 \pi f_{\pi}\right)\right)^{2} \log \left(\Lambda_{\chi} / m_{\pi}\right)$, which is consistent with the expected divergence of the isovector charge radius in the chiral limit $m_{\pi} \rightarrow 0$ [26].

- An important issue which needs to be addressed is that of the contributions of higher excited states. We have estimated these contributions with the help of the simpler NRCQM sum rule (7). In general, the approach to saturation depends on the specific potential adopted. For an harmonic oscillator the sum rule is saturated with the first P-wave states alone and for a particle in a spherical potential well these states contribute over $99 \%$ of the total. A similar behaviour is expected to be true of any other confining potential. On the other hand, in the hydrogen atom the lowest-lying 
P-wave states contribute $55 \%$ and the continuum states about $28 \%$ of the total [4].

- The r.h.s. of (12) is an increasing function of $\beta$, with a minimum at $\beta=1.93$ (for $\left.g^{2}=0.5\right)$. Therefore requiring equality of the two sides seems to favor values for $\beta$ of the order of $3 \mathrm{GeV}^{-1}$. However, the estimate of the P-wave contributions is still too crude to allow setting an useful constraint on $\beta$. In case that these contributions turn out to have been overestimated, the sum rule will be well satisfied with larger values of $\beta\left(4-6 \mathrm{GeV}^{-1}\right)$.

A discussion of the $\mathrm{SU}(3)$-breaking effects is now in order. $\mathrm{SU}(3)$ was needed to express the photoproduction cross-section $\sigma_{1 / 2}(\omega)$ in (4) in terms of the decay widths $\Gamma$ in (5). When it is broken, the relation between these two is not anymore a simple one: the former quantity only depends on the isovector properties of the excited state, whereas the latter contains both isovector and isoscalar contributions (unrelated to each other).

However, let us assume that the individual partial widths of all multipoles allowed in a given radiative decay $\Gamma=\sum_{i} \Gamma_{i}$ will be measured experimentally. Then it will be possible to eliminate the isoscalar transition matrix element between the same partial widths for the two members of the isospin doublet $\Gamma_{i}^{(u)}, \Gamma_{i}^{(d)}$. One obtains in this way

$$
\sigma_{1 / 2}(\omega)=\frac{\pi^{2}}{4} \sum_{\text {exc }}(2 J+1) \sum_{i} \frac{\left(\sqrt{\Gamma_{i}^{(u)}}-\sqrt{\Gamma_{i}^{(d)}}\right)^{2}}{|\vec{k}|^{3}} \delta(\omega-\delta M) .
$$

The determinations of the square roots must be chosen which satisfy $\sqrt{\Gamma_{i}^{(u)}}: \sqrt{\Gamma_{i}^{(d)}}=$ $2 / 3:-1 / 3$ in the $\mathrm{SU}(3)$-symmetric limit. With this choice (5) is recovered in the $\mathrm{SU}(3)$ limit. This method requires a good theoretical control over the signs of the matrix elements involved. Also from the experimental point of view the measurement of the different partial widths might appear to be very difficult. However, if successful, the subtraction in (13) will eliminate at the same time with the matrix element of the isoscalar light quark e.m. current, also the contribution from the heavy quark. This will extend the domain of applicability of the sum rule to the charm sector, for which the validity of the heavy mass expansion is less certain. 
Finally, we note that the methods of this paper can be used with little modification to obtain a sum rule for the pion couplings between heavy excited mesons and the ground state ones $B, B^{*}$. To derive it, consider the amplitude for forward scattering of pions on a $B$ meson. The assumption of an unsubtracted dispersion relation for the isospin-odd part of this amplitude plus knowledge of its low-energy limit gives an analog of the well-known Adler-Weisberger sum rule [2, 3]

$$
1-g^{2}=\frac{f_{\pi}^{2}}{\pi} \int_{m_{\pi}}^{\infty} \frac{\mathrm{d} \nu}{\nu^{2}} \sqrt{\nu^{2}-m_{\pi}^{2}}\left(\sigma\left(\pi^{-} B_{u} \rightarrow X\right)-\sigma\left(\pi^{+} B_{u} \rightarrow X\right)\right)
$$

On the l.h.s. $g$ is the $\mathrm{BB}^{*} \pi$ coupling defined as above; the integral on the r.h.s. runs over the inclusive cross-sections for $B_{u} \pi^{ \pm}$scattering with energy $\nu$. Just as in the case of the CR sum rule, the heavy resonances will contribute only to $\sigma\left(\pi^{-} B_{u} \rightarrow X\right)$, since the $\pi^{+} B_{u}$ state has isospin 3/2. Separating explicitly the contribution of the resonances from the continuum $\left(I_{\text {cont }}\right)$, the sum rule (14) can be written as

$$
1=2 \pi f_{\pi}^{2} \sum_{r e s}(2 J+1) \frac{\Gamma\left(B_{d}^{r e s} \rightarrow \pi^{-} B_{u}\right)}{\nu^{3}}+I_{\text {cont }} .
$$

Even without a detailed calculation one can see that $I_{\text {cont }}$ is positive, because $B_{u} \pi^{-}$has simply more available channels than $B_{u} \pi^{+}$. This observation can be used to produce a model-independent constraint on the couplings of the higher resonances:

$$
\begin{aligned}
& g^{2}+|h|^{2}+(0.07 \pm 0.01)+\cdots<1 \\
& \left(B^{*}\right) P_{1 / 2} \quad P_{3 / 2}
\end{aligned}
$$

The contribution of the $s_{\ell}^{\pi_{\ell}}=3 / 2^{+}$heavy mesons has been obtained from existing data on their decay widths [27,28]. On the other hand, the members of the $s_{\ell}^{\pi_{\ell}}=1 / 2^{+}$multiplet whose strong couplings are parametrized by $h$ (defined as in 29]) have not been observed experimentally due to their large width. The relation (16) gives an useful upper bound on $|h|:|h|^{2}<0.5$, where we neglected the small contribution from the other excited states and used $g^{2}=0.5$ as above. This agrees well with the recent QCD sum rule calculation of [30], who find $|h|^{2}=0.39 \pm 0.31$. For the total pionic widths of the $0^{+}$and $1^{+}$charmed states 
our result implies the upper bounds $\Gamma\left(0^{+}\right) \leq 328-560 \mathrm{MeV}$ and $\Gamma\left(1^{+}\right) \leq 110-250 \mathrm{MeV}$, corresponding to the mass values $m_{0^{+}}=m_{1^{+}}=2.3-2.4 \mathrm{GeV}$ 31].

\section{ACKNOWLEDGMENTS}

C.K.C. thanks Peter Lepage, Mark Wise and Tung-Mow Yan for discussion. His work is supported by the National Science Foundation. D.P. thanks Jürgen Körner and Karl Schilcher for useful discussions on the subject of this paper. He acknowledges a grant from the Deutsche Forschungsgemeinschaft (DPG). 


\section{REFERENCES}

$\dagger \quad$ Address after Jan. 1st: Technion, Haifa 32000, Israel.

[1] N. Cabibbo and L.A. Radicati, Phys. Lett. 19697 (1966).

[2] S. Adler, Phys. Rev. Lett. 141051 (1965); Phys. Rev.140 B736 (1965).

[3] W. Weisberger, Phys. Rev. Lett. 141047 (1965); Phys. Rev.143 1302 (1965).

[4] H. Bethe and E. Salpeter, Quantum mechanics of one- and two-electron atoms, Springer-Verlag, Berlin 1957.

[5] M.A. Beg, Phys. Rev. 1501276 (1966).

[6] K. Kawarabayashi and W.W. Wada, Phys. Rev. 1521286 (1966).

[7] S. Weinberg, in Lectures on Elementary Particles and Quantum Field Theory, 1970 Brandeis University Summer Institute on Theoretical Physics (M.I.T. Press, Cambridge, 1970), pp. 285-393

[8] P. Cho and H. Georgi, Phys. Lett. B296 408 (1992).

[9] J. Amundson et al., Phys. Lett. B296 415 (1992).

[10] H.Y. Cheng et al., Phys. Rev. D46 5060 (1992).

[11] A. de Rujula, H. Georgi and S.L. Glashow, Phys. Rev. D12 3589 (1975).

[12] M. Wise, Phys. Rev. D45 2188 (1992).

[13] G. Burdman and J.F. Donoghue, Phys. Lett. B280 287 (1992).

[14] H.Y. Cheng et al., Phys. Rev. D46 1148 (1992).

[15] T.M. Aliev et al., Phys. Lett. B334 169 (1994); METU-HE-15-95, hep-ph/9511290.

[16] P. Colangelo, F. De Fazio and G. Nardulli, Phys. Lett. B334 175 (1994); Phys. Lett. B339 151 (1994). 
[17] A.G. Grozin and O.I. Yakovlev, BUDKERINP-94-3, hep-ph/9401267.

[18] V.M. Belyaev et al., Phys. Rev. D51 6177 (1995).

[19] H.G. Dosch and S. Narison, PM-95-41, hep-ph/9510212.

[20] N. Isgur, D. Scora, B. Grinstein and M. Wise, Phys. Rev. D39 799 (1989).

[21] N. Isgur and D. Scora, Phys. Rev. D52 2783 (1995).

[22] M.A. Luty and R. Sundrum, Phys. Rev. D52 1627 (1995); Phys. Rev.D52 5202 (1995).

[23] F.J. Gilman and H.J. Schnitzer, Phys. Rev. 1501362 (1966)

[24] S.L. Adler and F.J. Gilman, Phys. Rev. 1561598 (1967)

[25] C.A. Dominguez and H. Moreno, Phys. Rev. D13 616 (1976)

[26] M.A. Beg and A. Zepeda, Phys. Rev. D6 2912 (1972).

[27] U. Kilian, J.G. Körner and D. Pirjol, Phys. Lett. B288 360 (1992).

[28] A. Falk and M. Luke, Phys. Lett. B292 119 (1992).

[29] A. Falk, Phys. Lett. B305 268 (1993).

[30] P. Colangelo, F. de Fazio, G. Nardulli, N. Di Bartolomeo and R. Gatto, hep-ph/9506207.

[31] S. Godfrey and N. Isgur, Phys. Rev. D32 189 (1985); S. Godfrey and R. Kokoski, Phys. Rev. D43 1679 (1991). 